\title{
The disparity of maternal childcare pressure and quality of life between children diagnosed with autism spectrum disorder and developmental delay in the Chinese culture context: Taiwan Birth Cohort pilot Study
}

For-Wey Lung

Calo Psychiatric Center

Bih-Ching Shu ( $\nabla$ forweylung@gmail.com )

National Cheng Kung University https://orcid.org/0000-0001-7697-1493

Tung-Liang Chiang

National Taiwan University

Shio-Jean Lin

Chi Mei Medical Center

Research article

Keywords: Autism spectrum disorder, Taiwan Birth Cohort Study, community-based study, family support, parental quality of life

Posted Date: April 3rd, 2020

DOI: https://doi.org/10.21203/rs.3.rs-19625/v1

License: (c) (1) This work is licensed under a Creative Commons Attribution 4.0 International License. Read Full License 


\section{Abstract}

Background: Many studies have shown that parents express higher stress when taking care of children with autism spectrum disorder (ASD). However, cultural context should be taken into consideration when investigating children's diagnosis and parental quality of life (QOL). Therefore, this study aimed to investigate the pathway relationship among parental QOL, child development, ASD and developmental delay (DD) risk at 3 years of age, and ASD or DD diagnosis 2.5 years later.

Methods: A birth cohort study database collected at age 3 and 5.5 years was used $(\mathrm{N}=1618)$. The Modified Checklist of Autism in Toddlers, Taiwan Birth Cohort Study Developmental Instrument, and the Short Form-36 was used to assess ASD risk, developmental condition (DD risk), and parental QOL, respectively.

Results: By structural equation modeling, an indirect influence was found between children's diagnosis and maternal health QOL. Mothers of children diagnosed with ASD perceived more family support, and those who perceived more family support had better health QOL. Conversely, mothers of children diagnosed with DD perceived higher childcare pressure, and mothers who perceived higher childcare pressure had worse health QOL.

Conclusions: The differences in mother's experience when their children are diagnosed with DD and ASD can only be found in pathway analysis, showing the importance of structural equation models. Healthcare personnel should take into consideration the cultural context of the families we encounter, and the vital role family support plays should be taken into consideration when interacting with families from collectivism cultures.

\section{Background}

Autism spectrum disorder (ASD) is characterized by the two core set features of impaired social communication and social interactions, and restricted and repetitive behavior and interests [1]. Many studies have shown that taking care of children with ASD increases stress levels in both parents [2,3], and higher levels of parental stress can contribute to lower quality of life (QOL) for parents of children with ASD [4]. Although both parents can be affected by the stress of raising a child with ASD, previous studies have shown inconsistent results in the level of stress and its effect on QOL for mothers and fathers. Dardas and Ahmad found that the parenting stress was elevated in both parents of children with ASD and was predictive of QOL $[4,5]$. However, since mothers are generally the core caregivers, other researchers have found that they have a higher level of stress than fathers, with increased rates of physical health problems and poorer QOL [6]. Mothers of children with developmental delay (DD) and intellectual disability also suffer greater stress and depression than those of typically developing children [7-9]. Meta-analysis also showed that parenting stress of parents of children with ASD or DD were higher, even when compared to parents of children with other clinical diagnosis [10]. Furthermore, a study in Vietnam found having a child with cognitive delay was the strongest predictor of maternal stress [11]. 
When an individual deems that environmental stressors have overwhelmed their resources, they engage coping mechanisms to restore functioning, however if their coping mechanisms are maladaptive or cannot meet the new demands, the outcome is stress [12]. Therefore, to understand the stress that parents experience, it is also important to understand the broader familial context and factors that may moderate the bidirectional effect between parental stress and child development [12]. Weiss emphasized the importance of emotional and social resources, showing that maternal positive marital adjustment, sense of accomplishment in parenting, social support, and higher educational achievement were protective factors for stress-related somatic symptoms [13]. Social support is a health-promoting resource, which has a direct positive effect on mental and physical health [14]. This may be especially important for parents of children with disabilities, since they may experience associative stigma, which is extension of stigma to those associated with the stigmatized individual [15]. This stigma may have a greater influence in Chinese society; a culture characterized by collectivism, prioritization of the group over the individual, and where the close links between individuals are emphasized. In a Chinese study, it was found that the stigma experienced by caregivers of children with intellectual disability was of greater concern for parents in China than it was in other cultures [16]. Therefore, mothers of children with ASD in the Chinese culture may need greater support than mothers in individualistic cultures.

Maternal education is also an important factor that has a more persistent and pervasive effect than parental mental health on child development [17]. Furthermore, children of mothers with higher education have better development than children of mothers with lower levels of education $[17,18]$. Therefore, in an investigation of child development, maternal level of education is an important confounding factor that needs to be controlled.

With all these matters in mind, a community-based study investigating the relationship between parental QOL and children with DD or ASD was conducted. Maternal pressure to take care of the child, level of education, and family support can affect the relationship between parental QOL, DD or ASD risk and diagnosis; however, this effect may be suppressed if not investigated [19].

Therefore, using structural equation modeling (SEM) in a stratified community-based sample in Taiwan, this study aimed to investigate the pathway relationship among parental QOL, level of education, child development, ASD and DD risk at 3 years of age, and ASD or DD diagnosis 2.5 years later. Moderating or meditating factors for maternal stresses including childcare pressure and family support were also included.

\section{Methods}

\section{Participants}

The dataset from the Taiwan Birth Cohort Pilot Study (TBCS-P) was used. The TBCS-P was a national birth cohort study that sampled infants born between November and December 2003 in Taiwan, with no exclusion criteria. Two-stage stratified random sampling was carried out. In the first stage, the primary 
sampling unit was cities and towns, and 29 of 365 townships were chosen using systematic random sampling. In the second stage, the selected primary sampling unit was strata by levels of urbanization and total fertility rate, which helped to obtain a better idea of the effect of urbanization on diagnosis. For instance, in the United States, estimated ASD prevalence was significantly higher in cities that had access to education sources compared with cities that relied solely on health sources to identify cases [20]. Similarly, urbanization disparity was found in the diagnostic rate for ASD in Taiwan [18], showing the importance of accessibility to resources. The final target sample of 2,048 babies was selected, resulting in an approximate sampling rate of $12 \%$. Three stages of data were collected: when the children were 6 , 18 and 36 months old. The dataset for the third stage, with 1,630 children at 36 months of age, was the one used for this study. At 5.5 years parents were asked whether their children had ever been diagnosed with ASD or DD. Children who participated in all four stages were included in this study, resulting in a sample size of 1,618 children. The protocol of this study was approved by the Institutional Review Board of a teaching hospital in Taiwan. Written informed consent was obtained from all participants included in the study.

The demographics of the infants and their parents are shown in Table 1. Most of the main caregivers who filled out the questionnaires were mothers $(n=1537,94.9 \%)$. A small percentage $(n=42,2.6 \%)$ was filled out by fathers. Of the 1,618 children, approximately half were male $(n=888,54.9 \%)$. The mean age of the fathers at childbirth was $37.87 \pm 5.34$ (range $21-60$ years), and the mean age of mothers was $33.92 \pm 4.84$ (range $22-50$ years).

\section{Materials}

Information was collected from the parent or main caregiver. After the parents agreed to participate at each stage of the study, a trained researcher visited their homes and conducted a structured interview, collecting data on all variables that may have affected child health and development. Typical development was assessed using the Taiwan Birth Cohort Study Developmental Instrument (TBCS-DI), and ASD risk was measured using the Modified Checklist of Autism in Toddlers (M-CHAT). Parental QOL was measured using the Taiwanese version of the SF-36 Health Survey.

M-CHAT is the most widely used and researched screening tool for ASD, and was originally developed for use as a primary screener in the general population [21]. It is a parent-report screening instrument that includes $23 \mathrm{yes/no} \mathrm{items} \mathrm{[21].} \mathrm{There} \mathrm{are} \mathrm{two} \mathrm{criteria} \mathrm{for} \mathrm{failing} \mathrm{the} \mathrm{M-CHAT:} \mathrm{(1)} \mathrm{failing} \mathrm{three} \mathrm{of} \mathrm{the} 23$ items, and (2) failing two of the six critical items. Those who fail in one of the two criteria are suspected of having ASD. The M-CHAT requires no parental or physician training, and is thus easy to use. The positive predictive value of the M-CHAT was 0.36 for screening of those in the general population aged 16-30 months [22].

The TBCS-DI is used as the screening instrument for DD. TBCS-DI is a parental report of children's daily performance. TBCS-DI is a culturally sensitive, easily comprehensible instrument, measuring child development in four dimensions: gross motor, fine motor, language, and social development. The 6,18 , 36 , and 66-month scales have been established, and the 36 month scale was used in this study, which 
consisted of 19 items: six gross motor, five fine motor, four language, and four social items [23-25]. The cutoff point of point of 52 for developmental delay was used in this study [26]. The TBCS-DI exhibited a Cronbach's alpha of $0.79 \mathrm{I}$, and the correlation among the four dimensions was between 0.38 and 0.61 [26].

The SF-36 was used to measure maternal and paternal individual QOL, and not family QOL. The SF-36 Health Survey is a 36-item self-report of the overall physical and mental health status of an individual, and the effect of their health status on everyday activities. The SF-36 Health Survey includes eight scales measuring eight domains, which can be summarized into two scores: physical health summary (physical component summary; PCS) and mental health summary (mental component summary; MCS). These two summary scores give an overall assessment of QOL related to physical and mental health conditions. The Taiwanese version of the SF-36 Health Survey was translated from the US version of the SF-36 Health Survey, developed by Ware and colleagues [27]. The Taiwanese version of the SF-36 Health Survey exhibits item-scale correlation coefficients of $0.40-0.83$, and an internal reliability of Cronbach's alpha > 0.70 for all scales [28], with higher scores implying better health conditions.

Maternal childcare pressure was measured by asking three questions. (1) "In the past year, have you felt a lot of mental pressure from taking care of this baby?" (2) "Do you feel a lot of time pressure from taking care of this baby?" (3) "Do you feel a lot of social pressure from taking care of this baby?". These items were answered in a five-point Likert scale of whether they experienced "very heavy pressure", "somewhat heavy pressure", "average pressure", "not much pressure", or "no pressure at all” in childcare. The ratings of these three items were combined to form the childcare pressure factor.

Similarly, family support was measured by asking the mother "Are you satisfied with the help your family provides when you encounter difficulties?" on a three-point Likert rating scale of "rarely", "sometimes", and "always".

\section{Statistical analysis}

The demographic distribution of the children and parents, with the numbers having been screened from the M-CHAT and TBCS-DI, was analyzed using SPSS 15.0 for Windows (Chicago, IL). Missing data were replaced using Bayesian analysis; an analytic approach that uses all the available information to produce a maximum likelihood estimate. Linear and logistic regression analysis was used to analyze the factors that would affect parental mental and physical QOL, typical child development, and risk of ASD. To investigate the specific ASD behavior demonstrated by the children causing stress to the parents, items in M-CHAT were also analyzed.

The pathway relationships of the variables that had a significant effect on parental QOL and child development were further investigated using SEM. All variables associated with parental QOL, and children's DD/ASD diagnosis were included to control for confounding variables. The AMOS 7.0 statistical software package (SPSS) was used for SEM analysis. SEM results are presented as $\chi^{2}$, $p$ value, comparative fit index (CFI), and adjusted goodness-of-fit index (AGFI). The significance of the $\chi^{2}$ value is 
judged by the $p$ value, and AGFI indicates the variance of the model. A model with a p value $>0.05, \mathrm{CFI}>$ 0.95 , root mean square error of approximation (RMSEA) $<0.08$, and AGFI $>0.9$ implies that the null model approximates the real structure.

Variables associated with parental QOL and children's DD/ASD diagnosis, including parental QOL, maternal childcare pressure, family support, level of education, child development, and ASD risk were all included in the regression and SEM analysis to control for confounding variables. To make the results easier to read and interpret, only the parsimonious models are shown, meaning that only statistically significant variables are presented.

\section{Results}

Using M-CHAT, two different criteria were used to screen for those who were at high risk for ASD (Table 1). Using the first criterion, failure on three of 23 items, 292 (18.0\%) of the children were shown to be at high risk of ASD, and 24 (1.5\%) were shown to be at high risk using the second criterion, of not passing two of the six critical questions. All the children screened using the second criterion were also screened for ASD in the first criterion, resulting in a final total of $292(18.0 \%)$ being screened as possibly having ASD. In addition, 519 (32.1\%) children were screened as possibly having DD using the TBCS-DI instrument at 36 months of age (Table 1 ). One hundred and thirty-one (8.1\%) children were screened as being at high risk of both DD and ASD. At age 5.5 years, five $(0.3 \%)$ children reported having been diagnosed with ASD, $26(1.6 \%)$ with DD, and of these, 1 had a concurrent diagnosis of ASD and DD.

The correlation among the investigated variables is shown in Table 2. Results showed that maternal and paternal QOL were statistically significantly correlated. Maternal level of education was positively correlated with childcare pressure $(r=0.11, p<0.001)$, and negatively correlated with ASD and DD risk $(r=$ $-0.11, p<0.001 ; r=-0.11, p<0.001)$. Family support was associated with ASD diagnosis $(r=0.06, p=$ $0.016)$, and childcare pressure with DD diagnosis $(r=0.07, p=0.009)$.

The maternal perceived childcare pressure and maternal QOL were compared between mothers of children at risk of DD, ASD, and/or diagnosed with DD, ASD and controls, as shown in Table 3. Statistically significant difference was only found in the physical QOL between mothers of children at high risk of $D D$ and those not at risk $(F=11.91, p=.001)$.

The parsimonious linear regression model of the demographics, and child development effect on parental mental and physical health QOL, are shown (Table 4). Higher maternal level of education was associated with lower maternal physical health QOL $(\beta=-0.09, p<0.001)$. In addition, being at higher risk for ASD (measured using M-CHAT), and family support were associated with better maternal physical health QOL $(\beta=0.06, p=0.023 ; \beta=0.07, p=0.007)$. In terms of maternal mental health $Q O L$, higher levels of education and childcare pressure were associated with lower maternal mental health $Q O L(\beta=-0.17, p=$ $0.007 ; \beta=-0.14, p<0.001)$. In contrast, the perception of more family support was associated with better maternal mental health QOL $(\beta=0.05, p=0.027)$. None of the factors analyzed predicted paternal QOL. 
SEM was used to investigate the pathway relationship between childcare pressure, parental health QOL, and child development. The model resulted in a p value of $0.137(>0.05)$, CFI of $0.996(>0.95)$, RMSEA of 0.013 (<0.08) and AGFI of 0.991 (>0.9) (Fig. 1). In the parental health QOL aspect, the SEM results were similar to the regression results. Mothers who perceived better family support, less childcare pressure or with lower levels of education, were associated with better mental health QOL $(\beta=0.06, p=0.018 ; \beta=-0.14$, $p<0.001 ; \beta=-0.06, p=0.012$ ). Correspondingly, mothers who perceived better family support or who had lower levels of education tended to have better physical health QOL $(\beta=0.07, p=0.045 ; \beta=-0.09, p<$ 0.001). The health QOL of fathers was only correlated with the health QOL of mothers, thus was removed from the model.

In terms of the child developmental aspect, mothers with higher levels of education were associated with being at lower risk of DD and ASD $(\beta=-0.11, p<0.001 ; \beta=-0.09, p<0.001)$. Both higher DD and ASD risks were associated with being diagnosed with DD or ASD at age 5.5 years (DD diagnosis: $\beta=0.11, p<$ $0.001 ; \beta=0.05, p=0.030 ;$ ASD diagnosis: $\beta=0.06, p=0.011 ; \beta=0.08, p=0.002$ ).

Bidirectional relationship between children's diagnosis and mother's perceived childcare pressure and family support was investigated. Interestingly, mothers of children diagnosed with DD perceived higher childcare pressure, on the other hand, mothers of children diagnosed with ASD perceived more family support $(\beta=0.07, p=0.006 ; \beta=0.06, p=0.015)$.

\section{Discussion}

Our community-based birth cohort study did not find statistically significant difference between the maternal level of childcare pressure among the ASD, DD and controls. Pathway analysis showed the diagnosis of children did not have a direct association with parental health QOL, instead an indirect influence was found. Mothers of children diagnosed with ASD perceived more family support, and those who perceived more family support had better health QOL. On the other hand, mothers of children diagnosed with DD perceived higher childcare pressure, and mothers who perceived higher childcare pressure had worse health QOL. In the demographics aspect, higher maternal level of education was associated with lower maternal QOL, but had a lower risk of DD or ASD diagnosis. Paternal health QOL was only associated with maternal QOL.

Our community-based pathway model found children of mothers diagnosed with ASD perceived more family support, and higher family support was associated with better health QOL. In addition, mothers of children diagnosed with DD perceived more childcare pressure compared to those not diagnosed with DD, but mothers of children diagnosed with ASD did not perceive higher childcare pressure compared to mothers of children without ASD diagnosis. This results differ from previous research which have generally found that caring for a child with ASD is associated with greater parenting stress, compared to families of a child with typical development and other disabilities [29]. We hypothesize that this difference is due to differences in previous studies were of clinical self-referred samples and cultural context. Previous parental stress research was selected from clinical samples, meaning those who 
experience more stress about their children's behavior problems, and it's the level of stress which motivates them to seek treatment for their child [10]. Studies have shown that children's behavior problem contribute more to parental stress than their children's cognitive delay [30,31], thus it is understandable parents from clinical sample would report higher stress compared to those in the community.

In the cultural aspect, Chinese culture is characterized by collectivism, emphasize relational interdependence and the close links among individuals, and the mode of relying on family and in-group. The advantage of interdependence is being able to benefit from a rich network of family, friends, and community [32], which supports our results of mothers of children diagnosed with ASD perceiving more family support. A study in Kuwait also found mothers of children diagnosed with ASD reported an association between the ability to enjoy life and receiving support from the family [33]. However, the disadvantage of vertical collectivism is the emphasis of obedience to authorities, therefore subordinating one's own goals to those of the in-group, and restricting the pursuit of individual happiness [34, 35], and placing greater importance on family priorities than on individual needs [36]. As a result, stigma experienced by family members as a consequence of being associated with a stigmatized relative [16] is of greater concern for parents in the Chinese culture compared to those in other cultures. A study in Vietnam also showed parents of children with cognitive delays perceived higher parenting stress when they experience higher level of stigma [37]. Tremendous effort has been placed in increasing the public health awareness of ASD in Taiwan, including public campaigns, parliament advocate for awareness, establishment of ASD parent groups, the growth in public knowledge of ASD decreases stigma toward the diagnosis [38]. On the other hand, DD being a more generalized diagnosis, is not discussed as much and does not receive as much public attention, thus higher pressure maybe perceived by parents of children with DD. With the increased public awareness on ASD, family support for ASD increases, however, mothers still experience pressure on childcare when their children are diagnosed with DD. The differences in mother's experience when their children are diagnosed with DD and ASD can only be found in pathway analysis, thus showing the importance of SEM.

Factors that affected maternal QOL included the following: mothers who perceived more family support were associated with better overall QOL, and mothers who perceived greater childcare pressure or were more highly educated were associated with lower health QOL. From the perspective of women's multiple social roles, family support and work both have an important influence on a mother's role as a parent [14, 39]. Social support has a buffering effect on both the mental and physical health of women, lowering their risk of depression and mortality, and giving them a greater likelihood of recovery from clinical illness [40]. Mothers of children with ASD have reported support from friends, family, and especially spouses as being important to help them cope emotionally [41].

Maternal level of education affected QOL, as well as children's risk for DD and ASD, as measured using TBCS-DI and M-CHAT. Children of mothers who were higher educated were associated with lower risk of DD or ASD. This is consistent with previous studies showing that children of mothers with higher education had better development $[17,18]$. Lung and colleagues further investigated the interactive effect of maternal level of education and location of residence with ASD diagnosis [18]. Children of mothers 
with higher than average level of education, and who lived in the city had a 2.37 times higher chance of being diagnosed with ASD, compared to children of mothers with a lower than average level of education and who loved in a rural area. However, the opposite phenomenon was found in M-CHAT screening for ASD risk, with parents of children who were screened out with a higher risk of ASD having a lower level of education [18].

Paternal QOL was not associated with child development; rather, it was associated with maternal QOL in both the mental and physical health aspects. This is consistent with a previous qualitative study that showed that fathers of children with ASD were not associated with their children's condition; however, their children's condition was associated with their partner's condition, which in turn affected them [41]. Another study also found that the stress perceived by fathers of children with ASD was not associated with child characteristics but was positively predicted by their partner's depression [42]. A review of stress and coping in parents of children with ASD found that maternal mental health predicted a positive paternal parenting experience [43], and their children's behavior does not affect fathers' directly, but rather through their spouses' stress [41]. Since mothers carry the main burden in terms of caregiving, their QOL and children's condition are more closely connected, while fathers serve as backup support for the mothers [41].

A limitation of this study is the small magnitude of effect. Since this is a community study, there is a spectrum of severity in the symptom of ASD included. Community based studies may differ from clinical studies, in that clinical cases have already sought help, thus they are generally more severe and difficult to recover. In the same line the sample size of those diagnosed is small ( 5 children with ASD diagnosis and 26 with DD diagnosis), resulting in the small variance. Furthermore, there are a broad array of factors which may effect parental QOL (such as children's genetic information, medical comorbidities, or whether other siblings have ASD/DD diagnosis), and children's risk and diagnosis for DD and ASD, only direct effect were investigated in this study. However, there maybe mediating factors, thus when only the direct effects are investigated the magnitude of effect may be lessened. Since this is the pilot of a longitudinal follow-up birth cohort study, the causality and associations of these factors can continuously be observed in the large birth cohort sample with more children diagnosed later follow-ups. The second limitation of this study was that all measurements were based on parental report, including perceived health QOL, maternal childcare pressure, and family support. We used the dataset from a national birth cohort study, with the aim to collect data on all variables that may affect the child health and development, thus only simplified inventories could be used to measure childcare pressure and family support, rather than clinician inventories. In addition, children's measures of development were also from parental reports, including those in the broadband development screening (TBCS-DI) and the narrowband ASD screening (M-CHAT) instruments. These screening instruments resulted in a high prevalence of ASD $(18 \%)$ and DD (32.1\%). However, it should be noted that previous studies have found that those screened as false positive performed substantially poorer academically than true negatives [44]. Furthermore, it has been shown that parental reports of their concerns are reflective of true problems $[45,46]$. It should be noted that the diagnostic rate found in our study is low compared to that in the US, where a prevalence of $2.24 \%$ for ASD and $3.57 \%$ for DD was found [47], therefore we hypothesize that some children screened 
out at high risk are still waiting to be diagnosed. A previous study found an urbanization and educational disparity in ASD diagnosis in Taiwan, with children of mothers with higher education and living in the city having a 2.37 times higher chance of being diagnosed with ASD compared to children of mothers wither lower level of education and living in rural areas [18]. Showing that parents and professionals in urban areas have higher awareness of the disorder [48], on the other hand, the lack of access to diagnostic services in rural areas leads to lower diagnosis [49]. This also reflects the need to raise awareness of ASD in Taiwan and prevent underdiagnosis.

\section{Conclusions}

We carried out a large stratified sample from the community, and found that under the Chinese cultural context, mothers of children diagnosed with ASD perceived more family support, and those who perceived more family support had better health QOL. On the other hand, mothers of children diagnosed with DD perceived more childcare pressure, and mothers who perceived more childcare pressure had worse health QOL. This differs from previous results of parents of children with ASD perceiving greater childcare pressure. Healthcare personnel should take into consideration the cultural context of the families we encounter. When most interventions are developed under individualism cultural context of utilizing infrastructure that reduce the necessity of relying on family and in-group, the vital role family support plays should be taken into consideration when interacting with families from collectivism cultures.

\section{Abbreviations}

AGFI

Adjusted goodness-of-fit indices

ASD

Autism spectrum disorder

CFI

Comparative fit index

DD

Developmental delay

M-CHAT

Modified Checklist of Autism in Toddlers

QOL

Quality of life

RMSEA

Foot mean square error of approximation

SEM

Structural equation modeling

SF-36 
Short Form 36

TBCS-DI

Taiwan Birth Cohort Study Developmental Instrument

TBCS-P

Taiwan Birth Cohort Pilot Study

\section{Declarations}

Ethics approval and consent to participate: The protocol of this study was approved by the Institutional Review Board of Taipei City Hospital, Taiwan (TCHIRB-1021105-E), and is in accordance with the Helsinki Declaration. Written informed consent was obtained from all participants included in the study.

Consent for publication: Not applicable

Availability of data and material: Data set can be applied from the Bureau of Health Promotion (Taiwan) http://www.bhp.doh.gov.tw/BHPnet/English/index.aspx

Competing interests: The authors declare that they have no competing interests

Funding: This study was funded by a grant from the Bureau of Health Promotion (DOH93-HP-1702, DOH94-HP-1802, DOH95-HP-1802, DOH96-HP-1702). The funders had no role in study design, data collection and analysis, decision to publish, or preparation of the manuscript. The authors had full access to all the data in the study and had final responsibility for the decision to submit for publication.

Authors' contributions: All authors designed the study. T.L. Chiang and S.J. Lin overlooked the sampling and experimental procedures. F.W. Lung and B.C. Shu undertook the statistical analysis and interpreted the analysis. F.W. Lung wrote the first draft of the manuscript. All authors contributed to and have approved the final manuscript.

Acknowledgements: The authors would like to thank all the researchers in the Taiwan Birth Cohort pilot Study team, and the families and children that participated in the study.

\section{References}

1. American Psychiatric Association. Diagnostic and Statistical Manual of Mental Disorders. 5th ed. Washington DC: American Psychiatric Association; 2013.

2. Davis NO, Carter AS. Parenting stress in mothers and fathers of toddlers with autism spectrum disorders: associations with child characteristics. J Autism Dev Disord. 2008;38:1278-91.

3. Hayes SA, Watson SL. The Impact of Parenting Stress: A Meta-analysis of Studies Comparing the Experience of Parenting Stress in Parents of Children With and Without Autism Spectrum Disorder. J Autism Dev Disord. 2013;43:629-42. 
4. Dardas L, Ahmad M. Quality of life among parents of children with autistic disorder: a sample from the Arab world. Res Dev Disabil. 2014;35:278-87.

5. Dardas L, Ahmad MM. Predictors of quality of life for fathers and mothers of children with Autistic Disorder. Res Dev Disabil. 2104;35:1326-33.

6. Yamada A, Kato M, Suzuki M, Suzuki M, Watanabe N, Akechi T, et al. Quality of life of parents raising children with pervasive developmental disorders. BMC Psychiatry. 2012;12:119.

7. Baker BL, Blacher J, Kopp CB, Kraemer B. Parenting children with mental retardation, Int Rev Res Ment Retard. 1997;20:1-45.

8. Shu BC, Lung FW, Chang YY. The mental health in mothers with autistic children: a case control study in southern Taiwan. Kaohsiung J Med Sci. 2000;16:308-14.

9. Singer GHS. Meta-analysis of comparative studies of depression in mothers of children with and without developmental disabilities. Am J Ment Retard. 2006;3:155-69.

10. Barroso NE, Mendez L, Graziano PA, Bagner DM. Parenting stress through the lens of different clinical groups: a systematic review \& meta-analysis. J Abnorm Child Psychol. 2018;46:0449-61.

11. Shin JY, Nhan NV. Predictors of parenting stress among Vietnamese mothers of young children with and without cognitive delay. J Intellect Dev Disabil.2009;34:17-26.

12. Shaw DS, Bell RQ. Developmental theories of parental contributors to antisocial behavior. J Abnorm Child Psychol. 1993;21:493-518.

13. Weiss MJ. Hardiness and social support as predictors of stress in mothers of typical children, children with autism, and children with mental retardation. Autism. 2002;6:115-30.

14. Repetti RL. Multiple roles. In: Blechman EA, Brownell KD, editors. Behavioral Medicine \& Women: a comprehensive handbook. New York: Guilford Press; 1998.

15. Farrugia D. Exploring stigma: Medical knowledge and the stigmatization of parents of children diagnosed with autism spectrum disorder. Sociol Health IIIn. 2009;31:1011-27.

16. Chiu MY, Yang, Wong FH, Li JH, Li J. Caregiving of children with intellectual disabilities in China-an examination of affiliate stigma and the cultural thesis. J Intellect Disabil Res. 2013;57:1117-29.

17. Lung FW, Shu BC, Chiang TL, Lin SJ. Parental mental health, education, age at childbirth and child development from six to 18 months, Acta Paediatr. 2009;98:834-41.

18. Lung FW, Chiang TL, Lin SJ, Shu BC. Urban and education disparity for autism spectrum disorders in Taiwan Birth Cohort Study. J Autism Dev Disord. 2017;47:599-606.

19. MacKinnon DP, Krull JL, Lockwood CM. Equivalence of the mediation, confounding and suppression effect. Prev Sci. 2000;1:173-81.

20. Autism and Developmental Disabilities Monitoring Network Surveillance Year 2008 Principal Investigators. Prevalence of Autism Spectrum Disorders - Autism and Developmental Disabilities Monitoring Network, 14 Sites, United States, 2008. MMWR Surveill Summ. 2012;61:1-19.

21. Robins DL, Fein D, Barton ML, Green JA. the modified checklist for autism in toddlers: An initial study investigating the early detection of autism and pervasive developmental disorders. J Autism Dev 
Disord. 2001;31:131-44.

22. Kleinman JM, Robins DL, Ventola PE, Pandey J, Boorstein HC, Esser EL, et al. The modified checklist for autism in toddlers: a follow-up study investigating the early detection of autism spectrum disorders. J Autism Dev Disord. 2008;38:827-39.

23. Lung FW, Chiang TL, Lin SJ, Shu BC. Developing and refining the Taiwan Birth Cohort Study (TB CS): Five years of experience. Res Dev Disabil. 2011;32:2697-703.

24. Lung FW, Shu BC, Chiang TL, Lin SJ. Efficient developmental screening instrument for 6- and 18month-old children in the Taiwan Birth Cohort Pilot Study. Acta Paediatr. 2008;97:1093-8.

25. Lung FW, Shu BC, Chiang TL, Lin SJ. Autism-risk screening in the first 3 years of life in Taiwan Birth Cohort Pilot Study. Res Autism Spectr Disord. 2011;5:1385-9.

26. Lung FW, Shu BC, Chiang TL, Lin SJ. Child developmental screening instrument from six to thirty-six months in Taiwan Birth Cohort Study. Early Hum Dev. 2010;86:17-

27. Ware JE Jr., Gandek B, Kosinski, Aaronson NK, Apolone G, Brazier J, et al. The equivalence of SF-36 summary health scores estimated using standard and country-specific algorithms in 10 countries: results from the IQOLA Project International Quality of Life Assessment. J Clin Epidemiol. 1998;51:1167-70.

28. Lu JFR, Tseng HM, Tsai YJ. Assessment of Health-related Quality of Life in Taiwan (I): Development and Psychometric Testing of SF-36 Taiwan Version. Taiwan Journal of Public Health 2003;22:50111.

29. Hayes SA, Watson SL. The impact of parenting stress: a meta-analysis of studies comparing the experience of parenting stress in parents of children with and without autism spectrum disorder. $J$ Autism Dev Disord. 2013;43:629-42.

30. Baker BL, Blacher J, Crnic KA, Edelbrock C. Behavior problems and parenting stress in families of three-year-old children with and without developmental delays. Am J Ment Retard. 2002;107:433-44.

31. Baker BL, Blacher J, Olsson Preschool children with and without developmental delay: behaviour problems, parents' optimism and well-being. J Intellect Disabil Res. 2005;49:575-90.

32. Waterman AS. The psychology of individualism. New York: Praeger; 1984.

33. Al-Kandari S, Alsalem A, Abohaimed S, Al-Orf F, Al-Zoubi M, Al-Sabah R, et al. Brief Report: Social Support and Coping Strategies of Mothers of Children Suffering from ASD in Kuwait. J Autism Dev Disord. 2017;47:3311-9.

34. Hsu FLK. Rugged individualism reconsidered. Knoxville: University of Tennessee Press; 1983

35. Triandis HC. Cultural syndromes and subjective well-being. in: Diener E, Suh EM, editors. Culture and subjective well-being. Cambridge MA: MIT Press; 2000.

36. Chao RK. Chinese and European American mothers' beliefs about the role of parenting in children's school success. J Cross Cult Psychol. 1996;27:403-

37. Shin J, Nhan NV, Crittenden KS, Hong HT, Flory M, Ladinsky J. Parenting stress of mothers and fathers of young children with cognitive delays in Vietnam. J Intellect Disabil Res.2006;50:748-60. 
38. Obeid R, Daou N, DeNigris D, Shane-Simpson C, Brooks PJ, Gillespie-Lynch K. A cross-cultural comparison of knowledge and stigma associated with autism spectrum disorder among college students in Lebanon and the United States. J Autism Dev Disord. 2015;45:3520-36.

39. Werner S, Shulman C. Does type of disability make a difference in affiliate stigma among family caregivers of individuals with autism, intellectual disability or physical disability? J Intellect Disabil Res. 2015;59:272-

40. Helstrom AW, Blechman EA. Parenting. in: Blechman EA, Brownell KD, editors. Behavioral Medicine \& Women: a comprehensive handbook. New York: Guilford Press; 1998.

41. Wills TA. Social Support. in: Blechman EA, Brownell KD, editors. Behavioral Medicine \& Women: a comprehensive handbook. New York: Guilford Press; 1998.

42. Gray DE. Gender and coping: the parents of children with high functioning autism. Soc Sci Med. 2003;56:631-42.

43. Hastings RP, Kovshoff H, Ward NJ, degli Espinosa F, Brown T, Remington B. Systems analysis of stress and positive perceptions in mothers and fathers of pre-school children with autism. $\mathrm{J}$ Autism Dev Disord. 2005;35:635-44.

44. Flippin M, Crais ER. The need for more effective father involvement in early autism intervention a systematic review and recommendations. J Early Interv. 2011;33:24-

45. Glascoe FP. Are over-referrals on developmental screening tests really a problem? Arch Pediatr Adolesc Med. 2001;1551:54-9.

46. Filipek PA, Accardo PJ, Ashwal S, Baranek GT, Cook EH Jr, Dawson G, et al. Practice parameter: Screening and diagnosis of autism: Report of the Quality Standards Subcommittee of the American Academy of Neurology and the Child Neurology Society. Neurology. 2000;55:468-79.

47. Zablotsky B, Black LI, Maenner MJ, Schieve LA, Blumberg SJ. Estimated Prevalence of Autism and Other Developmental Disabilities Following Questionnaire Changes in the 2014 National Health Interview Survey. Natl Health Stat Report. 2015;13:1-

48. Chen CY, Liu CY, Su WC, Huang SL, Lin KM. Urbanicity-related variation in help-seeking and services utilization among preschool-age children with autism in Taiwan. J Autism Dev Disord. 2008;38:48997.

49. Coo H, Ouellette-Kuntz H, Lam M, Yu CT, Dewey D, Bernier FP, et al. Correlates of age at diagnosis of autism spectrum disorders in six Canadian regions. Chronic Dis Inj Can. 2012;32:90-100.

\section{Tables}

Table 1. Demographics of children and their parents $(\mathrm{N}=1,618)$ 


\begin{tabular}{lr} 
Main caregiver who completed the interview & \\
Mother & $1537(94.9)$ \\
Father & $42(2.6)$ \\
Boys & $888(54.9)$ \\
Twins & $52(3.2)$ \\
Mothers working & $1128(66.8)$ \\
Maternal education & \\
Elementary school & $59(3.6)$ \\
High school & $896(55.4)$ \\
University/college & $613(37.9)$ \\
Graduate school & $597(55.4)$ \\
Paternal education & $50(3.1)$ \\
Elementary school & \\
\hline Graduate school & \\
\hline
\end{tabular}

\section{M-CHAT}

Criterion 1: 3/23 failed items

$292(18.0)$

Criterion 2: 2/6 failed items

$24(1.5)$

Fulfilling either one of the criteria

$292(18.0)$

TBCS-DI 36-mo developmental delay

519 (32.1)

\begin{tabular}{lr}
\hline Diagnosed with ASD before 5 years old & $5(0.3)$ \\
Diagnosed with DD before 5 years old & $26(1.6)$ \\
Diagnosed with ASD and DD before 5 years old & $1(<0.1)$ \\
\hline Variable (range) & Mean (SD) \\
\hline Paternal age at time of childbirth (21-60 yr) & $37.87(5.34)$ \\
\hline Maternal age at time of childbirth (22-50 yr) & $33.92(4.84)$ \\
\hline
\end{tabular}


Table 2. Correlation of parental QOL, maternal stress, level of education, social support, child DD and ASD risks and later diagnosis

\begin{tabular}{|c|c|c|c|c|c|c|c|c|c|c|c|}
\hline & Father Physical & Father & Mother & Mother Mental & Maternal & Childcare & Family & ASD & $\mathrm{DD}$ & ASD & $\mathrm{DD}$ \\
\hline & \multirow[t]{2}{*}{ QOL } & Mental & Physical & \multirow[t]{2}{*}{ QOL } & \multirow[t]{2}{*}{ education } & \multirow[t]{2}{*}{ pressure } & \multirow[t]{2}{*}{ support } & \multirow[t]{2}{*}{ risk } & \multirow[t]{2}{*}{ risk } & \multirow[t]{2}{*}{ diagnosis } & \multirow[t]{2}{*}{ diagnosis } \\
\hline & & QOL & QOL & & & & & & & & \\
\hline Father Physical & & $0.65^{* * *}$ & $0.15^{* * *}$ & $0.12^{* * *}$ & -0.01 & -0.01 & $-0.05^{*}$ & 0.03 & -0.02 & -0.02 & 0.04 \\
\hline \multicolumn{12}{|l|}{ QOL } \\
\hline Father Mental & - & - & $0.13^{* * *}$ & $0.18^{* * *}$ & -0.04 & -0.04 & -0.02 & 0.05 & $<0.01$ & $<-0.01$ & $0.06^{*}$ \\
\hline \multicolumn{12}{|l|}{ QOL } \\
\hline Mother Physical & - & - & - & $0.63 * * *$ & $-0.10^{* * *}$ & -0.01 & $0.07 * *$ & $0.07 * *$ & $<0.01$ & 0.05 & $<0.01$ \\
\hline \multicolumn{12}{|l|}{ QOL } \\
\hline Mother Mental & - & - & - & - & $-0.08^{* *}$ & $-0.15^{* * *}$ & $0.06^{*}$ & 0.05 & 0.03 & 0.01 & 0.02 \\
\hline \multicolumn{12}{|l|}{ QOL } \\
\hline Mother & - & - & - & - & - & $0.11^{* * *}$ & $<0.01$ & $-0.11^{* * *}$ & $-0.11^{* * *}$ & $<0.01$ & -0.03 \\
\hline \multicolumn{12}{|l|}{ education } \\
\hline Childcare & - & - & - & - & - & - & -0.01 & 0.03 & 0.04 & 0.04 & $0.07 * *$ \\
\hline \multicolumn{12}{|l|}{ pressure } \\
\hline Family support & - & - & - & - & - & - & - & 0.02 & -0.01 & $0.06^{*}$ & $<0.01$ \\
\hline ASD risk & - & - & - & - & - & - & - & - & $0.13 * * *$ & $0.09 * * *$ & $0.07 * *$ \\
\hline DD risk & - & - & - & - & - & - & - & - & - & $0.08 * *$ & $0.11 * * *$ \\
\hline
\end{tabular}

ASD: autism spectrum disorder; DD: developmental delay; QOL: quality of life. ${ }^{*} \mathrm{p}<0.05 ; * * \mathrm{p}<0.01 ; * * \mathrm{p}<0.001$

Table 3. Comparison of the quality of life and childcare pressure between mothers of children at risk or diagnosed with DD or ASD with controls 


\begin{tabular}{|c|c|c|c|}
\hline Comparison group & & $\mathrm{T}$ & $\mathrm{p}$ \\
\hline \multirow[t]{3}{*}{ ASD risk } & Childcare pressure & .73 & .394 \\
\hline & Maternal mental health QOL & 1.40 & .236 \\
\hline & Maternal physical health QOL & .01 & .936 \\
\hline \multirow[t]{3}{*}{ DD risk } & Childcare pressure & .01 & .933 \\
\hline & Maternal mental health QOL & 1.89 & .170 \\
\hline & Maternal physical health QOL & 11.91 & $.001^{* *}$ \\
\hline \multirow[t]{3}{*}{ ASD diagnosis } & Childcare pressure & 2.64 & .105 \\
\hline & Maternal mental health QOL & .92 & .338 \\
\hline & Maternal physical health QOL & .73 & .393 \\
\hline \multirow[t]{3}{*}{ DD diagnosis } & Childcare pressure & .04 & .852 \\
\hline & Maternal mental health QOL & .08 & .778 \\
\hline & Maternal physical health QOL & .13 & .716 \\
\hline
\end{tabular}

Table 4. Parsimonious linear regression model of demographics and child development effect on parental QOL

\begin{tabular}{llccc}
\hline Dependent variable & Independent variable & $\beta$ & $\mathrm{t}$ & $\mathrm{p}$ \\
\hline Maternal physical health QOL & Maternal education & -0.09 & -3.59 & $<0.001$ \\
& M-CHAT high risk for ASD & 0.06 & 2.28 & 0.023 \\
& Family support & 0.07 & 2.72 & 0.007 \\
& Maternal education & -0.07 & -2.70 & 0.007 \\
& Childcare pressure & -0.14 & -5.58 & $<0.001$ \\
& & & & \\
& Family support & 0.05 & 2.12 & 0.027 \\
\hline
\end{tabular}

ASD: autism spectrum disorder; M-CHAT: Modified Checklist of Autism in Toddlers; QOL: quality of life

\section{Figures}




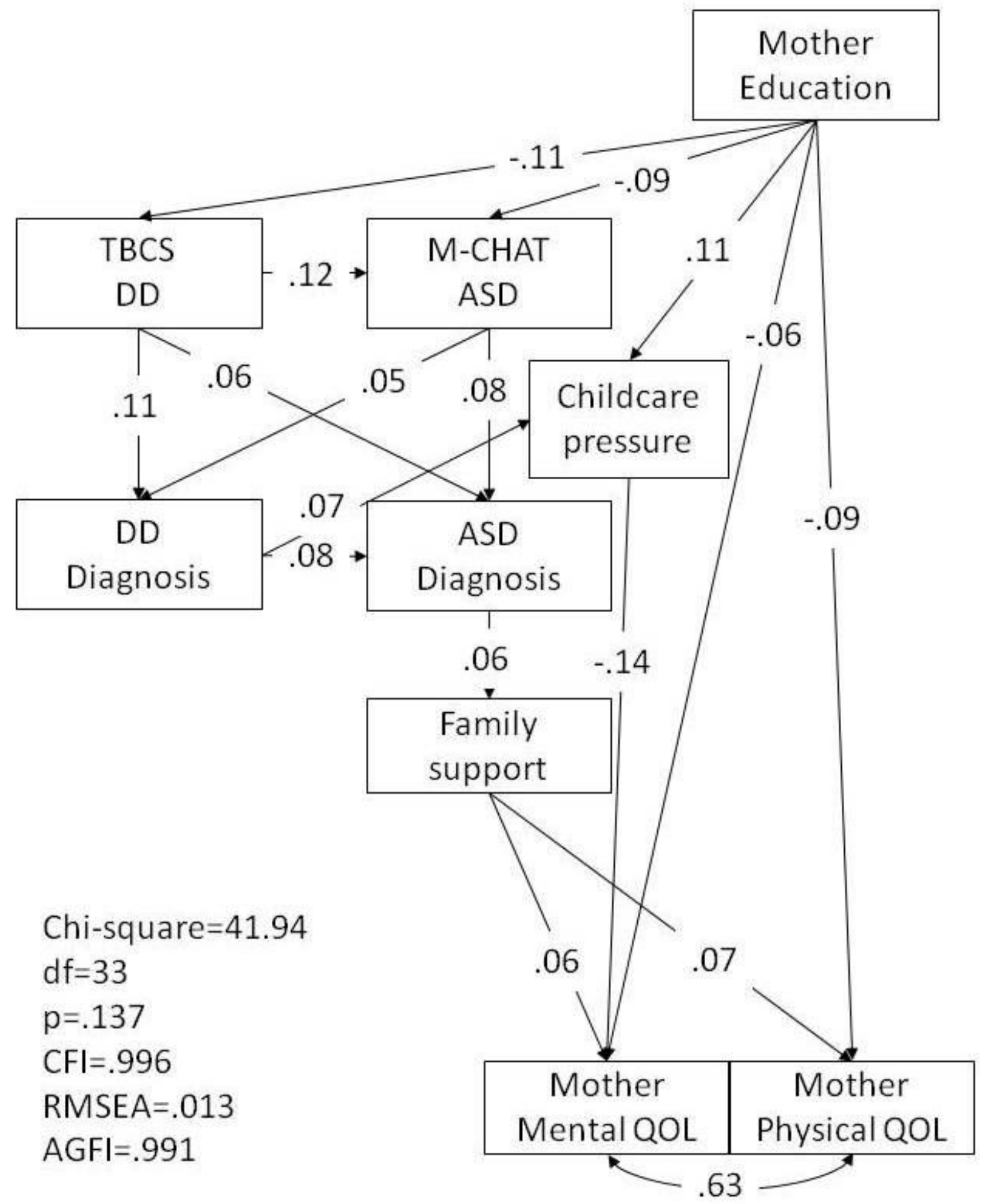

Figure 1

Pathway relationship among parental pressure, quality of life (QOL), the Taiwan Birth Cohort Study (TBCS) developmental instrument, and the Modified Checklist of Autism in Toddlers (M-CHAT) AGFI: adjusted goodness-of-fit indices; RMSEA: root mean square error of approximation; M-CHAT autism spectrum disorder and TBCS development delay dummy variable: 1, at risk, 0, not at risk 